\title{
UTILIDAD DE LOS RESCATES DE FAUNA: APORTES A LA HISTORIA NATURAL DE LOS MICROMAMÍFEROS EN LA LAGUNA DEL MAULE.
}

\author{
Jorge Mella Ávila \\ Cedrem Consultores, Padre Mariano 82, oficina 1003. Santiago, Chile. \\ E-mail: jorge.mella@,cedrem.cl
}

\begin{abstract}
RESUMEN
En el sector de la Laguna del Maule (Región del Maule), y como parte de un proyecto energético, se realizó un rescate de micromamíferos, en el verano y otoño de 2014. De dicho estudio se extrajo información de historia natural de las seis especies más abundantes. Se rescataron 440 ejemplares, siendo Phyllotis darwini la especie más abundante en los rescates, con 198 ejemplares $(48,10 \%$ del total), seguido de Abrothrix hirta, con 157 individuos (34,97\%). Al comparar las abundancias en los tres pisos altitudinales, se observan cambios en todas las especies. Así $P$. darwini, es más abundante y es la especie dominante en el piso inferior (1.400-1.500 msnm), disminuyendo gradualmente en los pisos superiores, mientras que el caso opuesto lo presenta $A$. hirta, cuyas capturas aumentan con la altitud, siendo dominante en el piso superior (2.100-2.200 msnm). Paynomys macronyx y Euneomys chinchilloides son especies más bien andinas en la zona central, ya que son escasas o ausentes a baja altura, y su abundancia relativa va aumentando con la altitud. Las hembras de $P$. darwini fueron de mayor tamaño y peso que los machos. En cuanto al uso de microhábitat, se observa un uso diferencial de cada microhábitat por rango altitudinal, con uso mayoritario por matorral en el piso bajo, mientras que en los pisos superiores se utilizan roqueríos y matorral hidrófilo. Casi todas las especies muestran flexibilidad por uso de los distintos microhábitats disponibles, excepto $P$. macronyx, especie que en los tres pisos altitudinales se observa en mayor proporción en el matorral hidrófilo, lo que estaría indicando preferencia. Este estudio es un ejemplo de la utilidad complementaria que pueden tener los rescates de fauna, para aportar al conocimiento de la ecología de los micromamíferos de Chile.
\end{abstract}

Palabras claves: Roedores, Abrothrix hirta, Paynomys macronyx, microhábitat, Cordillera de los Andes (36 S), Región del Maule.

\section{ABSTRACT}

Utility of fauna rescues: contributions to the natural history from small mammals of Laguna del Maule. In Maule's Lagoon (in the Maule Region), and as part of an energy project, a rescue of micro-mammals was carried out in the summer and autumn of 2014. From this study, information on the natural history of the six most abundant species was obtained. 440 specimens were rescued, with Phyllotis darwini being the most abundant species, with 198 specimens $(48.10 \%$ of the total), followed by Abrothrix hirta, with 157 individuals (34.97\%). When comparing the abundances in the three altitudinal floors, changes are observed in all species. Thus $P$. darwini, is more abundant and is the dominant species on the lower floor (1,400-1,500 masl), gradually decreasing on the upper floors, while the opposite case is presented by $A$. hirta, whose captures increase with altitude, being dominant on the upper floor (2,100-2,200 masl). Paynomys macronyx and Euneomys chinchilloides are rather andean species, since they are scarce or absent at low altitude, and their relative abundance increases with altitude. Females of $P$. darwini were larger and heavier than males. Regarding the use of microhabitats, a differential use of each microhabitat by altitude range was observed, with the majority being used scrub on the low floor, while on the upper floors rocks and hydrophilic scrub are used. Almost all the species show flexibility to the use of different available microhabitats, except $P$. macronyx, a species that is observed in a greater proportion in the hydrophilic scrub in the three altitudinal floors, which would indicate preference. This study is an example of the complementary utility that wildlife rescues can have, to contribute to the knowledge of the ecology of micro-mammals in Chile.

Key words: Rodents, Abrothrix hirta, Paynomys macronyx, microhabitat, Andes mountains $\left(36^{\circ} \mathrm{S}\right)$, Maule Region. 


\section{INTRODUCCIÓN}

En las últimas décadas, los rescates de fauna en Chile se han aplicado como una medida de mitigación de impacto ambiental, y se han enfocado básicamente para especies sensibles de baja movilidad (como reptiles y micromamíferos; Torres-Mura et al. 2014). En dichos rescates, se capturan los ejemplares de las especies foco (en las áreas que serán intervenidas por la ejecución de un proyecto particular), y posteriormente se liberan en áreas previamente definidas como sitios de relocalización.

Además de la utilidad práctica directa, al aumentar la probabilidad de sobrevivencia de parte de la población intervenida, dicha actividad de rescate tiene una utilidad complementaria indirecta (poco aprovechada hasta el momento), al aportar conocimiento sobre historia natural y/o antecedentes ecológicos de las especies involucradas, de las que en muchos casos, se han realizado escasos estudios específicos, como los reptiles chilenos (Mella 2020).

En el sector de la Laguna del Maule, Chile central, se desarrolló el proyecto energético denominado Central Hidroeléctrica Los Cóndores (aprobado ambientalmente en el año 2011). Como parte de las medidas de mitigación de impactos ambientales de dicho proyecto, en el año 2014 se realizó un rescate y relocalización de fauna (reptiles y micromamíferos), cuyo informe generó una enorme base de datos (debido el alto número de individuos rescatados, ver detalle en CEA 2014), del que se puede extraer información ecológicamente relevante, como la de reptiles (Mella 2020) y micromamíferos (que se presenta en este estudio). Sin embargo, se debe tener cautela con los análisis de tales estudios, ya que pueden existir limitaciones, restricciones y/o supuestos no explícitos, de modo que las extrapolaciones o generalizaciones resultantes consideren dichos factores.

Entre los micromamíferos presentes en el área de estudio, se encuentran varias especies de roedores, además de una especie de marsupial, Thylamys elegans (Iriarte 2008, Muñoz-Pedreros y Yáñez 2009, Teta y Pardiñas 2014). De dichas especies, son muy escasos los antecedentes ecológicos o de historia natural, con muy pocos estudios específicos, como ocurre con Paynomys macronyx (Thomas, 1894; antes conocida como Chelemys macronyx, ver revisión en D’Elía et al. 2020) y Euneomys chinchilloides (Waterhouse, 1839), especies registradas en el área.

Cabe indicar una nota taxonómica importante, en relación a la especie Abrothrix longipilis, indicada en el informe (CEA 2014) como una de las especies rescatadas en el sector. Teta \& Pardiñas (2014) señalan que Abrothrix longipilis (Waterhouse, 1837) es un complejo de al menos dos especies: A. longipilis sensu stricto, restringida al centro de Chile $\left(30^{\circ}\right.$ y $34^{\circ} \mathrm{S}$, lo que coincide con Palma et al. 2010) y A. hirta, presente en Chile y Argentina, y en Chile desde los $35^{\circ}$ al norte de Tierra del Fuego (criterio usado en Canto el al. 2017 y validado en D’Elía et al. 2020). Según dicha distribución (asociada a comparaciones morfométricas del cráneo y coloración general), la especie presente en nuestro estudio $\left(36^{\circ} \mathrm{S}\right.$, región del Maule), descrita como Abrothrix longipilis en el informe de rescate, corresponde a A. hirta.

En base a los antecedentes descritos, y restringidos a los parámetros registrados en la base de datos del informe de rescate de fauna, el objetivo de este estudio fue describir, para seis especies de roedores de la zona de Laguna del Maule: a) su abundancia y distribución altitudinal, b) la proporción de sexos y el tamaño corporal; y c) el uso de microhábitat. 


\section{MATERIALES Y MÉTODOS}

De la página del Servicio de Evaluación Ambiental (www.sea.gob.cl), se extrajo el informe público de rescate de fauna (CEA 2014) del Proyecto Central Hidroeléctrica Los Cóndores, localizado en el sector de la Laguna del Maule ( $\left.36^{\circ} \mathrm{S}\right)$, en la Región del Maule.

El rescate de micromamíferos se realizó entre el 23 de febrero y el 4 de abril de 2014, en 15 polígonos, localizados en tres rangos altitudinales: entre 1.400 a $1.500 \mathrm{msnm}$ (seis polígonos), entre 1.900 a $2.000 \mathrm{msnm}$ (seis polígonos) y entre 2.100 a $2.200 \mathrm{msnm}$ (tres polígonos). El esfuerzo de trampeo fue máximo en el piso intermedio, con 2.288 trampas-noche, seguido por el piso inferior (con 1.897 trampasnoche) y mínimo en el piso superior, con 522 trampas-noche, lo que se relaciona en parte con la superficie de los polígonos de rescate, las que varían entre 13,7 ha (piso superior) hasta 34,8 ha (piso inferior; Tabla 1). En total, se rescataron 449 ejemplares de nueve especies de roedores no fosoriales, de los que 440 individuos $(98,0 \%)$ corresponden a las seis especies más abundantes: Phyllotis darwini (Waterhouse, 1837), Abrothrix hirta, A. olivacea (Waterhouse, 1837), Octodon bridgesi Waterhouse, 1845, Paynomys macronyx y Euneomys chinchilloides. Otras especies registradas y rescatadas fueron Phyllotys xanthopygus (Waterhouse, 1837), Abrothrix andina (Philippi, 1858) y Oligoryzomys longicaudatus (Bennett, 1832), pero debido al bajo tamaño de muestra de cada especie (entre 1 a 4 individuos), fueron excluidos del análisis. La base de identificación de las especies, en particular las diferencias entre las dos especies de Phyllotis, no se detallan en el informe de rescate. Para las seis especies foco del estudio, se recopiló la información en detalle de los siguientes parámetros: abundancia por piso altitudinal, tamaño corporal (LE, desde el extremo del hocico hasta la base de la cola, en cm), largo de cola (LC, en cm), peso (en gr) sexo y edad (adultos y juveniles), además del microhábitat en que se registró cada ejemplar por cada piso altitudinal. Para cada especie, se definió un ejemplar juvenil basándose tanto en su inmadurez sexual como su peso, con valores menores al mínimo registrado en la literatura (ver revisión en Redford y Eisenberg, 1992; MuñozPedreros y Yáñez, 2009).

Se comparó la abundancia (absoluta y relativa) en cada uno de los tres rangos altitudinales. Para cada especie, la abundancia relativa se expresó como el cociente (en porcentaje) entre el número de individuos de la especie y el número total de individuos (de todas las especies presentes en cada piso altitudinal). Para el tamaño corporal (LE y peso), se realizó una comparación entre machos y hembras de cada especie (excluyendo los juveniles), mediante la prueba t de Student. Para comparar la proporción de sexos observada con la esperada $(\mathrm{M} / \mathrm{H}=1,0)$, se realizó una prueba de $\chi^{2}$. Las pruebas estadísticas consideraron un nivel de significancia de 5\% y se realizaron con el programa Statistica 7.0. Finalmente (y separados por cada piso altitudinal), para el uso de microhábitat, se cuantificó donde se registró cada individuo, en categorías generales como: matorral, matorral espinoso, matorral hidrófilo, matorral pajonal, matorral rocoso, pajonal, pedregal arenal, pedregal matorral, ribera de río y roquerío. En el informe de rescate no se especifica ni la definición de cada microhábitat ni las especies vegetales dominantes en cada formación.

\section{RESULTADOS}

\section{ABUNDANCIA Y DISTRIBUCIÓN ALTITUDINAL}

El tamaño de muestra total fue de 440 individuos de las seis especies más abundantes, las que representan un $98,0 \%$ del total (el 2,0\% restante corresponde a las tres especies menos abundantes, con nueve ejemplares en total). Phyllotis darwini fue la especie más abundante en los rescates, con 198 ejemplares (48,10\% del total), seguido de Abrothrix hirta, con 157 individuos (34,97\%). Las otras cuatro especies son mucho menos abundantes: Abrothrix olivacea $(\mathrm{n}=31 ; 6,90 \%)$, Octodon bridgesi $(\mathrm{n}=28$; $6,24 \%)$, Paynomys macronyx $(\mathrm{n}=16 ; 3,56 \%)$ y Euneomys chinchilloides $(\mathrm{n}=10 ; 2,23 \%)$. 
Al comparar las capturas por piso altitudinal, éstas son máximas en el piso inferior (con 242 capturas), disminuyendo a 142 en el piso intermedio y a 65 en el piso superior (Tabla 1). El éxito de captura fue similar en los pisos extremos, con valores cercanos al $12 \%$, mientras que fue menor en el piso intermedio, con $6,2 \%$ (Tabla 1 ).

Al comparar las abundancias en los tres pisos altitudinales, se observan cambios en todas las especies. Así $P$. darwini es más abundante y es la especie dominante en el piso inferior (1.400-1.500 msnm) con $61,2 \%$ (148 ejemplares), disminuyendo a $31,7 \%$ individuos en el piso intermedio (45 individuos) y a sólo 7,7\% en el piso más alto (cinco ejemplares; Tabla 1 y Figura 1). El caso opuesto lo presenta $A$. hirta, cuyas capturas son altas en todos los pisos, variando entre 39 a 62 ejemplares (Tabla 1), pero al observar sus abundancias relativas se observa un aumento creciente con la altitud: de $23,1 \%$ en el piso bajo, sube a 43,7\% en el piso intermedio y a $60 \%$ en el piso superior, donde es la especie dominante (Figura 1). A. olivacea es la tercera especie dominante en el piso inferior, con 12,0\% (29 capturas), pero baja a sólo 3,1\% en el piso superior (Tabla 1 y Figura 1). Las otras tres especies presentan bajas capturas, variando entre ninguno a 20 ejemplares por piso altitudinal (Tabla 1), aunque igualmente se observan patrones interesantes, como en O. bridgesi, más abundante en el piso intermedio ( $=20$ capturas; 14,1\%; Tabla 1 y Figura 1$)$, mientras que $P$. macronyx y E. chinchilloides son especies presentes a mayor altitud, ya que son escasas o ausentes a baja altura (Tabla 1), y su abundancia relativa va aumentando con la altitud, llegando a 10,8\% y $6,2 \%$ (respectivamente) en el piso superior (Figura 1).

\section{PROPORCIÓN DE SEXOS Y TAMAÑO CORPORAL}

Para Phyllotis darwini, la proporción de sexos (considerando sólo los adultos) fue de $\mathrm{M} / \mathrm{H}=1,24$ (no distinta de $1 ; \chi^{2}=2,15 ;$ g.l. $=1 ; \mathrm{p}>0,05$ ), y el tamaño promedio fue significativamente mayor en las hembras $(\mathrm{LE}=9,30 \mathrm{~cm})$ que en los machos $(\mathrm{LE}=8,62 \mathrm{~cm}$; $\mathrm{t}$ de Student $=3,45 ; \mathrm{p}<<0,05$; Tabla 2), lo que también se verificó en el peso: las hembras fueron significativamente más pesadas que los machos (51,9 gr versus 47,9 gr, respectivamente; $\mathrm{t}=2,01 ; \mathrm{p}<0,05$; Tabla 3 ). En el caso de $A$. hirta (la segunda especie más abundante), la proporción de sexos es $\mathrm{M} / \mathrm{H}=1,90$ (significativamente distinta de $1 ; \chi^{2}=14,30$; g.1. $=1 ; \mathrm{p}<<$ $0,05)$ y la LE promedio es similar entre machos $(8,00)$ y hembras $(7,84)$, sin diferencia significativa entre

Tabla 1. Abundancia absoluta de micromamíferos por rango altitudinal, en Laguna del Maule.

\begin{tabular}{|l|c|c|c|}
\hline \multicolumn{1}{|c|}{ ESPECIE } & \multicolumn{3}{|c|}{ RANGO ALTITUDINAL } \\
\hline \multirow{2}{*}{ P. darwini } & $\mathbf{1 4 0 0 - 1 5 0 0}$ & $\mathbf{1 9 0 0 - 2 0 0 0}$ & $\mathbf{2 1 0 0 - 2 2 0 0}$ \\
A. hirta & 148 & 45 & 5 \\
A. olivacea & 56 & 62 & 39 \\
O. bridgesi & 29 & 0 & 2 \\
P. macronyx & 4 & 20 & 4 \\
E. chinchilloides & 1 & 8 & 7 \\
\cline { 1 - 4 } Otros & 0 & 6 & 4 \\
\cline { 1 - 1 } TOTAL & 4 & 1 & 4 \\
\hline Superficie (ha) & 242 & 142 & 65 \\
\hline Trampas-noche & 34,8 & 30,54 & 13,72 \\
\hline Éxito de captura (\%) & 1.897 & 2.288 & 522 \\
\hline Cobertura vegetal* & 78,7 & 6,21 & 52,45 \\
\hline
\end{tabular}

Nota: $*=$ Cobertura promedio de los polígonos. 
ellos ( $\mathrm{t}=0,93 ; \mathrm{p}>0,30$; Tabla 2), al igual que los pesos, pero con tendencia a ser mayor en machos $(33,6 \mathrm{~g})$ que en hembras (31,1 gr; $\mathrm{t}=1,91 ; \mathrm{p}>0,05$; Tabla 3). Para A. olivacea la proporción de sexos es de $\mathrm{M} / \mathrm{H}=$ 0,81 (no distinta de $1 ; \chi^{2}=0,31 ;$ g.l. $=1 ; \mathrm{p}>>0,05$ ), y en promedio, los machos son similares en tamaño que las hembras (7,85 cm versus $8,49 \mathrm{~cm}$, respectivamente; $\mathrm{t}=1,45 ; \mathrm{p}>0,10$; Tabla 2$)$, al igual que en el peso (30,0 gr versus 30,9 gr, respectivamente; $\mathrm{t}=0,27 ; \mathrm{p}>0,70$; Tabla 3 ). En $O$. bridgesi (la especie de mayor tamaño), la proporción de sexos fue de $\mathrm{M} / \mathrm{H}=4,50$ (significativamente muy distinta de $1 ; \chi^{2}=8,91$; g.l. $=1 ; \mathrm{p}<<0,05)$, y en promedio, también los machos son similares a las hembras, tanto en longitud $(15,85$ $\mathrm{cm}$ versus 13,58 cm, respectivamente; $\mathrm{t}=1,73 ; \mathrm{p}>0,09$; Tabla 2$)$ como en peso (163,1 gr versus 122,5 gr, respectivamente; $\mathrm{t}=1,42 ; \mathrm{p}>0,10$; Tabla 3 ). Para P. macronyx, la proporción de sexos fue $\mathrm{de} \mathrm{M} / \mathrm{H}=2,00$ (no distinta de $1 ; \chi^{2}=1,67$; g.l. $=1 ; \mathrm{p}>0,05$ ), y en promedio, los machos son similares a las hembras, en longitud $(9,71 \mathrm{~cm}$ versus $9,82 \mathrm{~cm}$, respectivamente; $\mathrm{t}=0,14 ; \mathrm{p}>0,80$; Tabla 2$)$ y en peso (53,0 gr versus $51,0 \mathrm{gr}$, respectivamente; $\mathrm{t}=0,32 ; \mathrm{p}>0,70 ;$ Tabla 3). Finalmente, para E. chinchilloides, la proporción de sexos fue de $\mathrm{M} / \mathrm{H}=4,00$ (no distinta de $1 ; \chi^{2}=3,60$; g.l. $=1 ; \mathrm{p}>0,05$ ), y en promedio, los machos son significativamente menores en tamaño que las hembras $(10,00 \mathrm{~cm}$ versus $11,90 \mathrm{~cm}$, respectivamente; $\mathrm{t}=$ 2,89; $\mathrm{p}<0,05$; Tabla 2), lo que, sin embargo, no se ratifica en el peso, siendo similares ambos sexos $(55,0$ gr versus $65,0 \mathrm{gr}$, respectivamente; $\mathrm{t}=1,35 ; \mathrm{p}>0,20$; Tabla 3 ).

Comparando el largo de la cola entre sexos, la única especie que mostró diferencias significativas fue $A$. olivacea: en promedio, la cola de las hembras $(6,43 \mathrm{~cm})$ fue significativamente más larga que la de los machos (5,82 cm; $\mathrm{t}=3,30 ; \mathrm{p}<0,05$; Tabla 4).

Finalmente, la proporción de longitud de cola/cuerpo (LC/LE) para todas las especies (considerando los valores promedio de LC y LE) son las siguientes. Para $P$. darwini, dicha proporción es 1,19 en los machos y 1,14 en las hembras; para $A$. hirta, el cociente es 0,87 en machos y 0,89 en hembras; en $A$. olivacea, la proporción es 0,74 en los machos y 0,76 en las hembras; para $O$. bridgesi, dicho cociente es 0,69 en los machos y 0,70 en las hembras; en P. macronyx, la proporción es 0,50 en los machos y 0,45 en las hembras. Finalmente, en E. chinchilloides, la proporción de cola/cuerpo es 0,80 en los machos y 0,62 en las hembras, y que es la mayor diferencia observada entre los sexos.

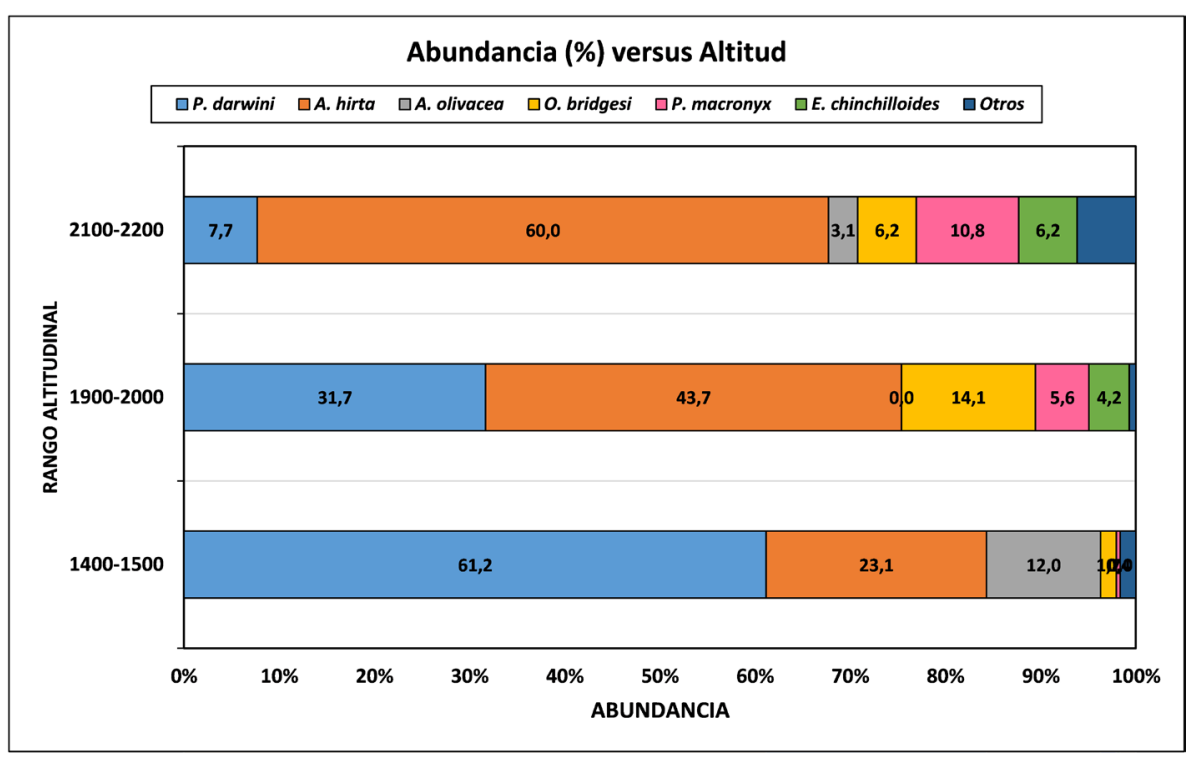

Figura 1: Abundancia relativa (\%) de micromamíferos por rango altitudinal, Laguna del Maule. 


\section{USO DE MICROHÁBITAT}

Considerando todas las especies, se observa un uso diferencial de cada microhábitat por rango altitudinal. Así, en el piso inferior (con cuatro microhábitats), la gran mayoría de los roedores (207 capturas, $85 \%$ ) se encuentra asociado a matorral, seguido por el matorral hidrófilo (18 observaciones; $8 \%$ ) y los roqueríos (15 registros; 6\%; Figura 2A). En el piso intermedio, con mayor diversidad de microhábitats (n $=8$ ), los roedores siguen usando el matorral, pero bajan a un 32\% (46 registros), muy similar al uso de roqueríos (42 observaciones; $30 \%$ ), a los que sigue el uso del matorral hidrófilo ( $\mathrm{n}=21 ; 15 \%)$, mientras los otros microhábitats presentan entre un 2\% a un 9\% (Figura 2B). Finalmente, en el piso superior (con cuatro tipos de microhábitats), los roedores se concentran en el matorral hidrófilo, con 39 registros (60\%), seguido por los roqueríos $(\mathrm{n}=13 ; 20 \%)$ y la ribera de río $(\mathrm{n}=10 ; 15 \%)$, mientras que el matorral presenta sólo 3 capturas $(5 \%$; Figura $2 \mathrm{C})$.

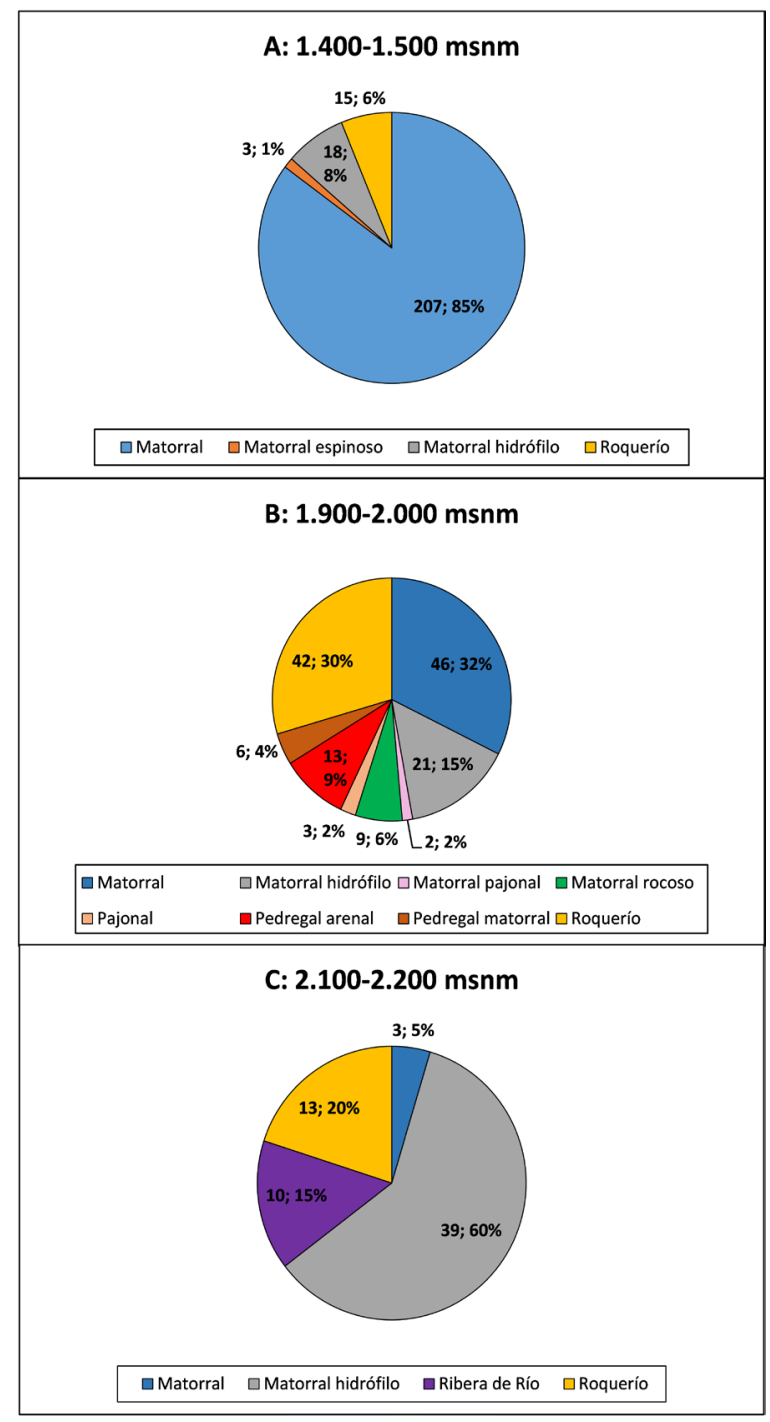

Figura 2: Abundancia relativa (\%) de micromamíferos por microhábitat, en cada piso altitudinal, Laguna del Maule. 


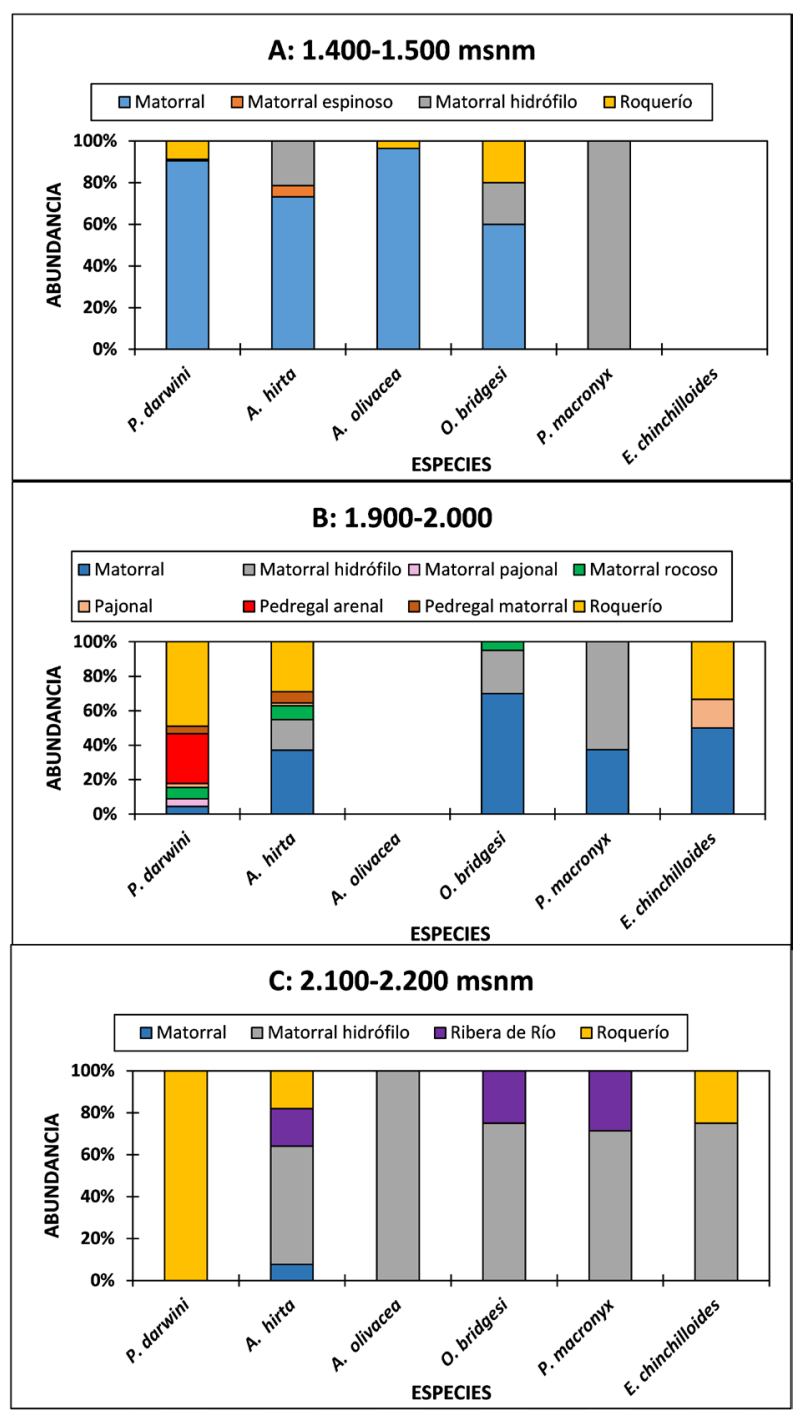

Figura 3: Abundancia relativa (\%) de cada especie de micromamífero por microhábitat, en cada piso altitudinal, Laguna del Maule.

Analizando cada especie de roedor, también se observan respuestas diferenciales. $P$. darwini se concentra principalmente $(90 \%)$ en el matorral en el piso inferior, usando el roquerío en el piso medio y en el piso superior, llegando en éste último a casi el 100\% de sus capturas (Figura 3). A. hirta usa mayoritariamente también el matorral en el piso inferior (más del 70\%), llegando a ser más capturado en el matorral hidrófilo en el piso superior (cerca del 50\%; Figura 3). A. olivacea es capturado mayoritariamente (más del 90\%) en matorral en el piso inferior, no aparece en las capturas en el piso intermedio y luego usa exclusivamente el matorral hidrófilo en el piso más alto, respuesta similar a $O$. bridgesi, quien también se observa en la ribera del río en el nivel superior (Figura 3). La especie menos flexible en el uso de microhábitat pareciera ser P. macronyx, quien en los tres pisos altitudinales se observa en mayor proporción en el matorral hidrófilo (Figura 3). Finalmente, E. chinchilloides cambia su uso mayoritario de matorral y roquerío en el piso medio a matorral hidrófilo en el piso superior (Figura 3). El detalle del total de las capturas en cada microhábitat para cada especie por rango altitudinal se muestra en el Apéndice 1. 
Tabla 2. Proporción de sexos (adultos) y tamaño corporal (Longitud Estándar = LE) de las especies de micromamíferos en Laguna del Maule.

\begin{tabular}{|c|c|c|c|c|c|c|c|}
\hline \multirow{2}{*}{ ESPECIE } & \multirow{2}{*}{ Sexo } & \multirow{2}{*}{$\mathrm{n}$} & & $\mathrm{LE}(\mathrm{cm})$ & \multirow{2}{*}{$\mathrm{t}$} & \multirow{2}{*}{$\begin{array}{l}\text { grados de } \\
\text { libertad }\end{array}$} & \multirow{2}{*}{$p$} \\
\hline & & & & Promedio +/- DE (Mín - Máx) & & & \\
\hline \multirow{2}{*}{ Phyllotis darwini } & Macho & 103 & \multirow{2}{*}{1,24} & $8,62+/-1,38(5,50-12,60)$ & \multirow{2}{*}{3,45} & \multirow{2}{*}{184} & \multirow{2}{*}{$0,0007 *$} \\
\hline & Hembra & 83 & & $9,30+/-1,27(5,50-12,50)$ & & & \\
\hline \multirow{2}{*}{ Abrothrix hirta } & Macho & 97 & \multirow{2}{*}{1,90} & $8,00+/-1,18(5,20-10,30)$ & \multirow{2}{*}{0,93} & \multirow{2}{*}{146} & \multirow{2}{*}{0,350} \\
\hline & Hembra & 51 & & $7,84+/-1,00(5,00-10,50)$ & & & \\
\hline \multirow{2}{*}{ Abrothrix olivacea } & Macho & 13 & \multirow{2}{*}{0,81} & $7,85+/-0,94(6,30-9,50)$ & \multirow{2}{*}{1,451} & \multirow{2}{*}{27} & \multirow{2}{*}{0,158} \\
\hline & Hembra & 16 & & $8,49+/-0,98(6,60-10,00)$ & & & \\
\hline \multirow{2}{*}{ Octodon bridgesi } & Macho & 18 & \multirow{2}{*}{4,50} & $15,85+/-2,34(8,70-19,90)$ & \multirow{2}{*}{1,726} & \multirow{2}{*}{20} & \multirow{2}{*}{0,099} \\
\hline & Hembra & 4 & & $13,58+/-2,61(10,30-16,50)$ & & & \\
\hline \multirow{2}{*}{ Paynomys macronyx } & Macho & 10 & \multirow{2}{*}{2,00} & $9,71+/-1,29(7,90-12,20)$ & \multirow{2}{*}{0,136} & \multirow{2}{*}{13} & \multirow{2}{*}{0,894} \\
\hline & Hembra & 5 & & $9,82+/-1,82(7,00-11,30)$ & & & \\
\hline \multirow{2}{*}{ Euneomys chinchilloides } & Macho & 8 & \multirow{2}{*}{4,00} & $10,00+/-0,89(9,30-11,50)$ & \multirow{2}{*}{2,889} & \multirow{2}{*}{8} & \multirow{2}{*}{$0,020^{*}$} \\
\hline & Hembra & 2 & & $11,90+/-0,14(11,80-12,00)$ & & & \\
\hline
\end{tabular}

Abreviaturas: DE = Desviación Estándar; Mín = Mínimo; Máx = Máximo.

Tabla 3. Peso (en gramos, sólo adultos) de las especies de micromamíferos en Laguna del Maule.

\begin{tabular}{|c|c|c|c|c|c|}
\hline \multirow{2}{*}{ ESPECIE } & \multirow{2}{*}{ Sexo } & Peso (gr) & \multirow{2}{*}{$\mathrm{t}$} & \multirow{2}{*}{$\begin{array}{c}\text { grados de } \\
\text { libertad }\end{array}$} & \multirow{2}{*}{$p$} \\
\hline & & Promedio +/- DE (Mín - Máx) & & & \\
\hline \multirow{2}{*}{ Phyllotis darwini } & Macho & $47,9+/-13,5(25,0-80,0)$ & \multirow{2}{*}{2,005} & \multirow{2}{*}{184} & \multirow{2}{*}{$0,046^{*}$} \\
\hline & Hembra & $51,9+/-13,4(25,0-85,0)$ & & & \\
\hline \multirow{2}{*}{ Abrothrix hirta } & Macho & $33,6+/-7,6(20,0-60,0)$ & \multirow{2}{*}{1,910} & \multirow{2}{*}{146} & \multirow{2}{*}{0,058} \\
\hline & Hembra & $31,1+/-7,8(20,0-55,0)$ & & & \\
\hline \multirow{2}{*}{ Abrothrix olivacea } & Macho & $30,0+/-11,0(15,0-55,0)$ & \multirow{2}{*}{0,266} & \multirow{2}{*}{27} & \multirow{2}{*}{0,792} \\
\hline & Hembra & $30,9+/-8,0(20,0-50,0)$ & & & \\
\hline \multirow{2}{*}{ Octodon bridgesi } & Macho & $163,1+/-52,1(50,0-270,0)$ & \multirow{2}{*}{1,415} & \multirow{2}{*}{20} & \multirow{2}{*}{0,172} \\
\hline & Hembra & $122,5+/-50,4(55,0-175,0)$ & & & \\
\hline \multirow{2}{*}{ Paynomys macronyx } & Macho & $53,0+/-11,1(30,0-65,0)$ & \multirow{2}{*}{0,321} & \multirow{2}{*}{13} & \multirow{2}{*}{0,753} \\
\hline & Hembra & $51,0+/-11,9(40,0-70,0)$ & & & \\
\hline \multirow{2}{*}{ Euneomys chinchilloides } & Macho & $55,0+/-9,6(40,0-70,0)$ & \multirow{2}{*}{1,352} & \multirow{2}{*}{8} & \multirow{2}{*}{0,213} \\
\hline & Hembra & $65,0+/-7,1(60,0-70,0)$ & & & \\
\hline
\end{tabular}

Abreviaturas: DE = Desviación Estándar; Mín= Mínimo; Máx = Máximo. 


\section{DISCUSIÓN}

\section{RIQUEZA Y COMPOSICIÓN}

De los micromamíferos registrados en los rescates, la composición de especies concuerda parcialmente con lo observado en zonas precordilleranas cercanas de la región del Maule, como en las localidades del Valle de Teno, Maitenes y Longaví (Reise y Venegas, 1987). Dichos autores registraron 11 especies en los sitios señalados, de las que seis especies concuerdan con nuestro estudio ( $P$. darwini, ambos Abrothrix, O. longicaudatus y $O$. bridgesi, además de Ctenomys maulinus, especie presente pero no rescatada). Las otras especies observadas por Reise y Venegas (1987) pero no capturadas en nuestro caso fueron Thylamys elegans, Dromiciops gliroides, Loxodontomys micropus, Aconaemys fuscus y Rattus norvegicus.

En el caso de Thylamys elegans, a pesar de ser una especie frecuente en varios ambientes de la zona centro-norte, es una especie poco abundante y de hábitos trepadores (Iriarte et al., 1989, Jiménez et al. 1992, Palma, 1997), conducta relativamente similar a D. gliroides y Loxodontomys micropus (Muñoz-Pedreros y Yáñez, 2009), características que explicarían en parte su ausencia en las capturas. Para Aconaemys fuscus y Spalacopus cyanus (otra especie presente en la región), sus conductas fosoriales podrían explicar su ausencia en las capturas, ya que prácticamente no caen en trampas Sherman, puestas a nivel del suelo. En el caso de Rattus norvegicus (y otros roedores exóticos presentes en la zona central), puede que la ausencia de capturas se explique porque la zona en estudio se encuentra lejos de centros poblados, donde dichas especies son más frecuentes y abundantes.

Tabla 4. Longitud de cola (LC) de las especies de micromamíferos en Laguna del Maule.

\begin{tabular}{|c|c|c|c|c|c|}
\hline \multirow{2}{*}{ ESPECIE } & \multirow{2}{*}{ Sexo } & $\mathrm{LC}(\mathrm{cm})$ & \multirow{2}{*}{$\mathrm{t}$} & \multirow{2}{*}{$\begin{array}{c}\text { grados de } \\
\text { libertad }\end{array}$} & \multirow{2}{*}{$p$} \\
\hline & & Promedio +/- DE (Mín - Máx) & & & \\
\hline \multirow{2}{*}{ Phyllotis darwini } & Macho & $10,30+/-1,64(6,00-13,80)$ & \multirow{2}{*}{1,766} & \multirow{2}{*}{184} & \multirow{2}{*}{0,0790} \\
\hline & Hembra & $10,72+/-1,52(4,20-13,40)$ & & & \\
\hline \multirow{2}{*}{ Abrothrix hirta } & Macho & $6,99+/-1,13(3,40-9,00)$ & \multirow{2}{*}{0,036} & \multirow{2}{*}{146} & \multirow{2}{*}{0,970} \\
\hline & Hembra & $7,00+/-0,96(4,50-9,50)$ & & & \\
\hline \multirow{2}{*}{ Abrothrix olivacea } & Macho & $5,82+/-0,48(5,00-6,60)$ & \multirow{2}{*}{3,305} & \multirow{2}{*}{27} & \multirow{2}{*}{$0,0027^{*}$} \\
\hline & Hembra & $6,43+/-0,50(5,70-7,40)$ & & & \\
\hline \multirow{2}{*}{ Octodon bridgesi } & Macho & $10,99+/-2,42(9,50-20,00)$ & \multirow{2}{*}{1,194} & \multirow{2}{*}{20} & \multirow{2}{*}{0,246} \\
\hline & Hembra & $9,50+/-1,00(8,00-10,00)$ & & & \\
\hline \multirow{2}{*}{ Paynomys macronyx } & Macho & $4,88+/-0,75(3,70-6,30)$ & \multirow{2}{*}{1,265} & \multirow{2}{*}{13} & \multirow{2}{*}{0,228} \\
\hline & Hembra & $4,38+/-0,65(3,50-5,20)$ & & & \\
\hline \multirow{2}{*}{ Euneomys chinchilloides } & Macho & $8,01+/-1,51(5,30-9,90)$ & \multirow{2}{*}{0,532} & \multirow{2}{*}{8} & \multirow{2}{*}{0,609} \\
\hline & Hembra & $7,40+/-0,99(6,70-8,10)$ & & & \\
\hline
\end{tabular}

Abreviaturas: $\mathrm{DE}=$ Desviación Estándar; Mín = Mínimo; Máx = Máximo. 


\section{ABUNDANCIA Y DISTRIBUCIÓN ALTITUDINAL}

Comparadas con otros registros en la zona centro-norte, la abundancia dominante de $P$. darwini en nuestro estudio concuerda con lo observado en San Carlos de Apoquindo (33 $23^{\prime} \mathrm{S}$, Iriarte et al. 1989), Aucó (31³0’ S, Jiménez et al. 1992) y Fray Jorge (30³8’, Jaksic et al. 1993). La segunda especie numéricamente importante en este estudio, $A$. hirta, muestra un patrón similar en el sur del país, ya que es una de las especies más abundantes en la Picada ( $41^{\circ} \mathrm{S}$, Patterson et al. 1989, descrita como A. longipilis). En relación a $O$. longicaudatus, especie registrada pero muy escasa en este estudio, su baja abundancia contrasta notoriamente con lo observado en la zona central, donde Iriarte et al. (1989) y a una altitud de $1.200 \mathrm{msnm}$, la registran como la segunda especie más abundante, después de $P$. darwini. Esta diferencia se explicaría por la distinta altitud entre ambos estudios, ya que en Laguna del Maule se capturaron $O$. longicaudatus sólo en el piso inferior (1.400 a $1.500 \mathrm{msnm}$ ), y no en los pisos más altos, lo que concuerda con su distribución altitudinal, la que llega a un máximo de 2.000 msnm (Muñoz-Pedreros y Yáñez, 2009). Otro factor explicativo es que la abundancia de O. longicaudatus varía enormemente según la productividad estacional, como ocurre con las denominadas "ratadas" en el sur de Chile (ver revisión en Redford y Eisenberg, 1992), por lo que es probable que en el área de estudio, en otra estación o año más productivo, esta especie sea mucho más abundante.

Los patrones contrastantes de abundancia relativa altitudinal de $P$. darwini (dominante a baja altitud) y $A$. hirta (dominante a mayor altura) pudieran explicarse por la probable disponibilidad de microhábitats diferentes en cada rango altitudinal, la posible preferencia diferencial de las especies por los distintos microhábitats, restricciones fisiológicas diferenciales (ej. preferencias térmicas) u otras causas. Ambas especies presentan un rango altitudinal muy similar, desde el nivel del mar hasta los $2.000 \mathrm{msnm}(A$. hirta) y 2.200 msnm ( $P$. darwini; Muñoz-Pedreros y Yáñez, 2009). En base a este último antecedente, es interesante destacar las capturas de $A$. hirta en el piso más alto (entre $2.100 \mathrm{msnm}$ y $2.200 \mathrm{msnm}$ ), lo que constituye un nuevo límite superior altitudinal para esta especie.

Los registros altitudinales de P. macronyx y E. chinchilloides, escasas o ausentes a baja altura y con abundancia relativa creciente con la altitud, son valiosos antecedentes para estas especies poco conocidas. Por una parte, se confirma que son especies poco abundantes, cuantificando entre $6 \%$ y $10 \%$ su abundancia máxima en el piso superior estudiado, y por otra parte, se ratifican como especies más bien andinas: para $P$. macronyx, su distribución altitudinal en la zona central se encuentra sobre los $1.000 \mathrm{msnm}$ (no detallando su altitud máxima; Muñoz-Pedreros y Yáñez, 2009), y como es una especie fosorial, es posible que el método de captura utilizado para su captura (trampas Sherman), puede estar sesgando los resultados. Para $E$. chinchilloides, en la zona central se ha registrado entre los $2.300 \mathrm{msnm}$ a los $3.000 \mathrm{msnm}$ (Muñoz-Pedreros y Yáñez, 2009). Comparado con dichos registros de literatura, nuestros antecedentes aportan nuevos límites altitudinales: para E. chinchilloides, su presencia en el piso intermedio, entre $1.900 \mathrm{msnm}$ a $2.000 \mathrm{msnm}$, constituye un nuevo mínimo altitudinal (al menos $400 \mathrm{~m}$ más abajo que el registro histórico), mientras que el registro de . macronyx en el piso superior $(2.200 \mathrm{msnm})$ es un nuevo valor máximo altitudinal.

\section{PROPORCIÓN DE SEXOS Y TAMAÑO CORPORAL}

De las seis especies analizadas en detalle, $A$. hirta, y $O$. bridgesi mostraron una proporción de sexos significativamente distinta de 1:1. Curiosamente, en ambos casos la proporción no es menor a 1,0, como se podría esperar de especies territoriales (como en el caso de guanacos y vicuñas, con un macho con un haren de varias hembras; Muñoz-Pedreros y Yáñez, 2009), sino que se registra mayor cantidad de machos que hembras $(M: H>1,0)$, lo que se puede asociar a artefactos de muestreo, como la heterogeneidad de capturas asociadas a sexo (Simonetti, 1986). Dicho autor indicó que los micromamíferos de la zona central muestran heterogeneidad de recapturas, y al menos cuatro especies (A. longipilis, A. olivacea, P. darwini y 
O. longicaudatus) muestran respuestas poblacionales diferentes. Así, mientras la mayoría de los individuos evaden las trampas (más notorio en $O$. longicaudatus y $P$. darwini), una proporción baja de las poblaciones de cada especie muestran preferencia por la recaptura, lo que dependería de varios factores, como sexo, edad y estatus social (ej. machos adultos capturados más frecuentemente que las hembras y juveniles; Simonetti, 1986).

Los tamaños corporales (en tamaño y peso) de las especies observadas en Laguna del Maule están entre los rangos registrados en la literatura (Muñoz-Pedreros y Yáñez, 2009; Redford y Eisenberg, 1992). Cabe destacar los registros de longitud y peso de $A$. hirta, con una media que varía entre $7,84 \mathrm{~cm} \mathrm{y} 8,00 \mathrm{~cm}$ de LE y 31,1 gr a 33,6 gr, para hembras y machos respectivamente. Comparado con A. longipilis (su especie hermana), A. hirta es significativamente más pequeña: los ejemplares de $A$. longipilis de la zona central (Santiago) miden 12,9 cm de LE y pesan una media de 62,5 gr (Muñoz-Pedreros y Yáñez, 2009). Estos antecedentes morfométricos complementan las diferencias morfológicas señaladas como características diagnósticas originales, basadas en medidas del cráneo y en coloración general (Teta y Pardiñas, 2014). Los valores aparentemente bajos de $A$. hirta comparados con los de otras poblaciones podría explicarse por un probable sesgo de la toma directa de la medida de LE, lo que no está detallado en el informe de rescate.

Comparando el tamaño de machos y hembras, sólo en $P$. darwini existe diferencia significativa, siendo las hembras más grandes y pesadas que los machos. Para A. longipilis y A. hirta, se ha mostrado que existen diferencias de tamaño entre los sexos (Gallardo et al. 1988, Teta \& Pardiñas 2014), lo que no concuerda con nuestro caso, en que ni la longitud ni el peso son distintos entre macho y hembra. Es probable que parte de las diferencias entre sexos sea explicado por la preferencia o evasión de las capturas (Simonetti 1986), factor ya discutido.

Comparando el largo de la cola entre sexos, la única especie que mostró diferencias significativas fue $A$. olivacea, donde la cola de las hembras es más larga que la de los machos (en valores absolutos). Sin embargo, al analizar las proporciones relativas (promedio de LC/LE), dichas diferencias se minimizan (0,74 y 0,76$)$. Comparando el cociente LC/LE entre ambos sexos para todas las especies, la mayor diferencia observada es en E. chinchilloides, aunque tales valores pueden estar sesgado por el bajo tamaño de muestras (con sólo dos hembras). Se desconocen antecedentes que comparen largo de la cola de ambos sexos de los roedores chilenos (Iriarte, 2008; Muñoz-Pedreros y Yáñez, 2009).

Finalmente, los cocientes LC/LE entregan valores cuantitativos (avalados por alto número de muestras), que pueden complementar los registros cualitativos de la descripción de las especies (Redford y Eisenberg, 1992; Iriarte, 2008; Muñoz-Pedreros y Yáñez, 2009). En el caso de P. darwini (descrito cualitativamente como de cola más larga que el cuerpo), dicha proporción de la cola es entre un $14 \%$ a un 19\% más larga que el cuerpo; para $A$. hirta (descrito como de cola más corta que el cuerpo), la cola varía entre un $11 \%$ a un $13 \%$ más corta que el cuerpo; en $A$. olivacea (también señalado como de cola más corta que el cuerpo), la proporción de cola oscila entre un $24 \%$ y un $26 \%$ más corta que el cuerpo); para O. bridgesi (descrito como que la cola es de un $44-46 \%$ de la longitud total, Muñoz-Pedreros y Yáñez, 2009), el valor varía entre 30 a 31\% de cola más corta que el cuerpo, lo que corresponde a un $41 \%$ de la longitud total (muy similar a lo ya descrito); en P. macronyx (descrito simplemente como de cola corta), la proporción de cola varía entre un 50\% y un 55\% más corta que el cuerpo. Estos datos pueden ser utilizados para discriminar especies similares (como las diferencias efectivamente encontradas entre $A$. hirta versus A. olivacea), y pudieran replicarse para determinar si hay diferencias entre especies y/o subespecies de géneros diversos, muy difíciles de discriminar morfológicamente (como Phyllotis spp.). 


\section{USO DE MICROHÁBITAT}

En este estudio, los roedores utilizan diferentes microhábitats, probablemente dependiendo en gran medida de la distinta disponibilidad en los tres pisos altitudinales, lo que se asocia a los tipos de vegetación. Según Luebert y Pliscoff (2017), el piso inferior de altitud corresponde al piso subandino de matorral bajo de altitud, donde la asociación característica corresponde a Matorral bajo mediterráneo andino de Chuquiraga oppositifolia y Discaria articulata, mientras que en la parte alta, la asociación característica del piso vegetacional corresponde a Matorral bajo mediterráneo andino de Laretia acaulis y Berberis empetrifolia, y al piso andino-inferior, con pajonal de Festuca thermarum y Senecio leucophyton.

En el piso inferior (con baja diversidad de microhábitats), los roedores usan mayoritariamente el matorral ( $85 \%$ de las capturas), mientras que en el piso intermedio, con mayor diversidad de microhábitats, los roedores diversifican su uso de microhábitats, usando además de matorrales, roqueríos y matorral hidrófilo, ambiente con uso dominante en el piso superior. La mayoría de las especies utilizan el matorral y muestran flexibilidad de uso de otros ambientes, como roqueríos (en $P$. darwini) y matorral hidrófilo (en A. hirta, A. olivacea y $O$. bridgesi). La especie que muestra menor flexibilidad de uso de ambientes es $P$. macronyx, la que en los tres pisos altitudinales se observa en mayor proporción en el matorral hidrófilo, independiente de su disponibilidad, lo que estaría indicando una preferencia por dicho microhábitat.

El uso de ambientes cerrados por los roedores en la Laguna del Maule, como matorrales y roqueríos, concuerda con lo registrado en el matorral centro-chileno, donde los micromamíferos utilizan con mayor frecuencia los espacios cercanos a arbustos y rocas, y menos frecuentemente los espacios abiertos entre los arbustos, lo que se explicaría al menos por dos factores: la presencia de alimento y la evasión de los depredadores (Simonetti y Otaíza, 1982; Simonetti, 1989). El microclima y las respuestas termorreguladoras asociadas a distintos microhábitats parecieran no estar asociados a la selección de microhábitats, al menos para $P$. darwni y A. hirta (Ebensperger y Simonetti 1996), las dos especies más abundantes en nuestro estudio.

En el matorral centro-chileno, $P$. darwini no muestra diferencias entre el uso de espacio abierto versus cerrado (Jaksic et al. 1981), por lo que dicha especie estaría usando los microhábitats en la misma proporción que su disponibilidad ambiental, lo que concordaría con lo observado en este estudio, en que esta especie cambia su uso de hábitats en los distintos pisos altitudinales (de matorral a los roqueríos). En contraste, A. longipilis en la misma zona del matorral central (Jaksic et al. 1981), muestra mayor uso por espacios cerrados, lo que podría asociarse al uso mayoritario de matorrales, lo que concuerda con el uso mayoritario de dicho ambiente en el sur por A. hirta (Valdivia, Murúa y González, 1979) y en los distintos pisos de altura en nuestro caso, desde matorrales en el piso bajo, a los matorrales hidrófilos en el piso de mayor altitud. Además, dicha especie se asocia a ambientes boscosos sucesionales tardíos, a diferencia de A. olivacea, más relacionado con ambientes sucesionales tempranos (más abiertos, Murúa et al. 1996).

Este estudio es sólo un ejemplo de la utilidad complementaria que pueden tener los rescates de fauna, al agregar nuevo conocimiento de la historia natural de especies poco conocidas, como $P$. macronyx y E. chinchilloides, así como especies recientemente reconocidas como distintas, como Abrothrix hirta (para la que se indican nuevos antecedentes morfométricos). Se sugiere revisar otros estudios similares, para aportar al conocimiento de otras especies de roedores (en otras regiones, como en el norte de Chile), o ampliarla a otros grupos (como reptiles, Mella 2020), los que poseen escasos estudios específicos.

\section{AGRADECIMIENTOS}

Agradezco a Cedrem Consultores la oportunidad de redactar esta publicación. A dos revisores anónimos, que ayudaron a mejorar este artículo. 


\section{REFERENCIAS BIBLIOGRÁFICAS}

CANTO, J., M. SALDARRIAGA y J. YÁÑEZ. 2017 Estudio craneométrico en Abrothrix hirta (Thomas, 1895) (Rodentia: Cricetidae): una aproximación desde la morfometría geométrica. Boletín del Museo Nacional de Historia Natural, Chile, 66(1): 101-123.

CEA 2014 Rescate y relocalización de fauna y flora asociada al Proyecto Hidroeléctrico Los Cóndores. Informe técnico, (Centro de Ecología Aplicada, CEA) 305 págs.

D'ELÍA, G., J. CANTO, G. OSSA, L. DARSI, E. BOSTELMANN, A. IRIARTE, L. AMADOR, M. QUIROGACARMONA, N. HURTADO, R. CADENILLAS y L. VALDEZ. 2020 Lista actualizada de los mamíferos vivientes de Chile. Boletín del Museo Nacional de Historia Natural, Chile, 69(2): 67-98.

EBENSPERGER, L.A. y J.A. SIMONETTI 1996 Microclimate and microhábitat selection in nocturnal rodents of central Chile: a test between biophysical and experimental approaches. Mammalia, 60(2): 195-209.

GALLARDO, M.H., G. AGUILAR y O. GOICOECHEA. 1988 Systematics of sympatric cricetid Akodon (Abrothrix) rodents and their taxonomic implications. Medio Ambiente9: 65-74.

IRIARTE, A. 2008 Mamíferos de Chile. Lynx ediciones, Barcelona, España. 420 págs.

IRIARTE, A. y J.A. SIMONETTI. 1986 Akodon andinus (Philippi, 1858): visitante ocasional del matorral esclerófilo centro-chileno. Noticiario Mensual, Museo Nacional de Historia Natural, Chile 311: 6-7.

IRIARTE, A., L.C. CONTRERAS y F.M. JAKSIC. 1989 A long-term study of a small mammal assemblage in the central Chilean matorral. Journal of Mammalogy, 70: 79-87.

JAKSIC, F.M. y J. YÁÑEZ.1979 Tamaño corporal de los roedores del distrito mastozoológico santiaguino. Noticiario Mensual del Museo Nacional de Historia Natural, Chile. 271: 3-4.

JAKSIC, F.M., J.L. YÁÑEZ y E.R. FUENTES. 1981 Assessing a small mammal community in central Chile. Journal of Mammalogy, 62(2): 391-396.

JAKSIC, F.M., P.L. MESERVE, J.R. GUTIÉRREZ y E.L. TABILO. 1993 The components of predation on small mammals in semiarid Chile: preliminary results. Revista Chilena de Historia Natural, 66: 305-321.

JIMÉNEZ, J., P. FEINSINGER y F.M. JAKSIC. 1992 Spatiotemporal patterns of an irruption and decline of small mammals in northcentral Chile. Journal of Mammalogy, 73: 356-364.

KELT, D.A. 1993 Irenomys tarsalis. Mammalian Species, $N^{\circ}$ 447:1-3.

LUEBERT, F. y P. PLISCOFF. 2017 Sinopsis bioclimática y vegetacional de Chile. Editorial Universitaria, Santiago, Chile. Segunda Edición. 381 págs.

MELLA, J.E. 2020 Aportes a la historia natural de los reptiles en la Laguna del Maule, otra utilidad de los rescates de fauna. Boletín Chileno de Herpetología 7: 27-33.

MUÑOZ-PEDREROS, A. y J. YÁÑEZ (Eds.) 2009. Mamíferos de Chile. Segunda Edición. CEA Ediciones, Valdivia. $571 \mathrm{pp}$.

MURÚA, R. y L.A. GONZÁLEZ. 1979 Distribución de roedores silvestres con relación a las características del hábitat. Anales del Museo Nacional de Historia Natural, Valparaíso, Chile, № 12: 69-75.

MURÚA, R., L.A.GONZÁLEZ y C. COFRÉ. 1996 Latitudinal variation of habit components in two species of Abrothrix (Rodentia: Cricetidae), in Chile. Medio Ambiente, 13(1): 3-10.

PALMA, R.E. 1997 Thylamys elegans. Mammalian Species, $N^{\circ}$ 572:1-4.

PALMA, R.E., R.A. CANCINO y E. RODRIGUEZ-SERRANO. 2010 Molecular systematics of Abrothrix longipilis (Rodentia: Cricetidae: Sigmodontinae) in Chile. Journal of Mammalogy 91:1102-1111.

PATTERSON, B., P.L. MESERVE y B.K. LANG. 1989 Distribution and abundance of small mammals along an elevational transect in temperate rainforests of Chile. Journal of Mammalogy, 70(1): 67-78.

REDFORD, K.H. y J.F. EISENBERG. 1992 Mammals of the Neotropics. The Southern Cone. Volume 2: Chile, Argentina, Uruguay, Paraguay. The University of Chicago Press, Chicago, 430 págs.

REISE, D. y W. VENEGAS. 1987 Catálogo de registros, localidadesy biotopes del trabajo de investigación acerca de los pequeños mamíferos de Chile y Argentina. Gayana, Zoología, 51(1-4): 103-130.

SIMONETTI, J. 1986 Heterogeneity of recaptures in Chilean small mammals. Revista Chilena de Historia Natural, 59(1): 55-63. 
SIMONETTI, J. 1989 Microhabitat use by small mammals in central Chile. Oikos, 56: 309-318.

SIMONETTI, J. y R. OTAÍZA. 1982 Ecología de micromamíferos de Chile central: Una revisión. Publicación Ocasional del Museo Nacional de Historia Natural, Chile, 38: 61-103.

TETA, P. y U. PARDIÑAS. 2014 Variación morfológica cualitativa y cuantitativa en Abrothrix longipilis (Cricetidae, Sigmodontinae). Mastozoología Neotropical, 21(2): 291-309.

TORRES-MURA, J.C., E. RIVEROS-RIFFO y V. ESCOBAR-GIMPEL. 2014 Guía técnica para implementar medidas de rescate/relocalización y perturbación controlada. Servicio Agrícola y Ganadero, Santiago, 45 pp. https:// www.sag.gob.cl/ambitos-de-accion/gestion-ambiental/133/publicaciones. 
Apéndice 1: Abundancia absoluta de micromamíferos por tipo de hábitat, en cada piso altitudinal.

A: $1.400-1.500 \mathrm{msnm}$

\begin{tabular}{|l|c|c|c|c|}
\hline \multirow{2}{*}{ ESPECIE } & \multicolumn{4}{|c|}{ HÁBITAT } \\
\cline { 2 - 5 } & Matorral & $\begin{array}{c}\text { Matorral } \\
\text { espinoso }\end{array}$ & $\begin{array}{c}\text { Matorral } \\
\text { hidrófilo }\end{array}$ & Roquerío \\
\hline P. darwini & 134 & 0 & 1 & 13 \\
\hline A. hirta & 41 & 3 & 12 & 0 \\
\hline A. olivacea & 28 & 0 & 0 & 1 \\
\hline O. bridgesi & 3 & 0 & 1 & 1 \\
\hline P. macronyx & 0 & 0 & 1 & 0 \\
\hline E. chinchilloides & 0 & 0 & 0 & 0 \\
\hline Otros & 1 & 0 & 3 & $\mathbf{1 5}$ \\
\hline TOTAL & $\mathbf{2 0 7}$ & $\mathbf{3}$ & $\mathbf{1 8}$ & \\
\hline
\end{tabular}

B: 1.900-2.000 msnm

\begin{tabular}{|l|c|c|c|c|c|c|c|c|}
\hline \multirow{2}{*}{ ESPECIE } & \multicolumn{9}{|c|}{ HÁBITAT } \\
\cline { 2 - 10 } & Matorral & $\begin{array}{c}\text { Matorral } \\
\text { hidrófilo }\end{array}$ & $\begin{array}{c}\text { Matorral } \\
\text { pajonal }\end{array}$ & $\begin{array}{c}\text { Matorral } \\
\text { rocoso }\end{array}$ & Pajonal & $\begin{array}{c}\text { Pedregal } \\
\text { arenal }\end{array}$ & $\begin{array}{c}\text { Pedregal } \\
\text { matorral }\end{array}$ & Roquerío \\
\hline$P$. darwini & 2 & 0 & 2 & 3 & 1 & 13 & 2 & 22 \\
\hline A. hirta & 23 & 11 & 0 & 5 & 1 & 0 & 4 & 18 \\
\hline A. olivacea & 0 & 0 & 0 & 0 & 0 & 0 & 0 & 0 \\
\hline O. bridgesi & 14 & 5 & 0 & 1 & 0 & 0 & 0 & 0 \\
\hline P. macronyx & 3 & 5 & 0 & 0 & 0 & 0 & 0 & 0 \\
\hline E. chinchilloides & 3 & 0 & 0 & 0 & 1 & 0 & 0 & 2 \\
\hline Otros & 1 & 0 & 0 & 0 & 0 & 0 & 0 & 0 \\
\hline TOTAL & $\mathbf{4 6}$ & $\mathbf{2 1}$ & $\mathbf{2}$ & $\mathbf{9}$ & $\mathbf{3}$ & $\mathbf{1 3}$ & $\mathbf{6}$ & $\mathbf{4 2}$ \\
\hline
\end{tabular}

C: 2.100-2.200 msnm

\begin{tabular}{|l|c|c|c|c|}
\hline \multirow{2}{*}{\multicolumn{1}{|c|}{ ESPECIE }} & \multicolumn{4}{c|}{ HÁBITAT } \\
\cline { 2 - 5 } & Matorral & $\begin{array}{c}\text { Matorral } \\
\text { hidrófilo }\end{array}$ & $\begin{array}{c}\text { Ribera de } \\
\text { Río }\end{array}$ & Roquerío \\
\hline P. darwini & 0 & 0 & 0 & 5 \\
\hline A. hirta & 3 & 22 & 7 & 7 \\
\hline A. olivacea & 0 & 2 & 0 & 0 \\
\hline O. bridgesi & 0 & 3 & 1 & 0 \\
\hline P. macronyx & 0 & 5 & 2 & 0 \\
\hline E. chinchilloides & 0 & 3 & 0 & 1 \\
\hline Otros & 0 & 4 & 0 & 0 \\
\hline TOTAL & $\mathbf{3}$ & $\mathbf{3 9}$ & $\mathbf{1 0}$ & $\mathbf{1 3}$ \\
\hline
\end{tabular}

